\title{
Isoproterenol Increases BIS and Arousal during Catheter Ablation for Atrial Fibrillation
}

\author{
O’Neill DK ${ }^{*}$, Aizer $\mathrm{A}^{2}$, Bloom MJ ${ }^{3}$, Kline RP ${ }^{4}$, Chinitz $\mathrm{L}^{5}$, Linton $\mathrm{PL}^{6}$ and Blanck $\mathrm{TJ}^{7}$
}

${ }^{1}$ Assistant Professor of Anesthesiology, Associate Director of Adult Off-Site Anesthesiology Services, New York University School of Medicine, New York University Langone Medical Center, New York, United States ${ }^{2}$ Assistant Professor of Medicine, Assistant Professor of Medicine and Cardiology, Associate Director of Cardiac Electrophysiology, Heart Rhythm Center, New York University School of Medicine, New York University Langone Medical Center, New York, United States

${ }^{3}$ Associate Professor of Anesthesiology, Director of Perioperative Technology, Co-Director of Neuroanesthesiology, New York University School of Medicine, New York University Langone Medical Center, New York, United States ${ }^{4}$ Senior Research Scientist, Department of Anesthesiology, New York University School of Medicine, New York University Langone Medical Center, New York, United States

${ }^{5}$ Professor of Medicine, Director of Cardiac Electrophysiology, Heart Rhythm Center, New York University School of Medicine, New York University Langone Medical Center, New York, United States

${ }^{6}$ Assistant Professor of Anesthesiology, Director of Adult Off-Site Anesthesiology Services and Endoscopy, New York University School of Medicine, New York University Langone Medical Center, New York, United States ${ }^{7}$ Professor of Anesthesiology, Professor, Department of Neuroscience Institute, New York University School of Medicine, New York University Langone Medical Center, New York, United States

*Corresponding author: O’Neill DK, Assistant Professor of Anesthesiology, Associate Director of Adult Off-Site Anesthesiology Services, New York University School of Medicine, New York University Langone Medical Center, 550 First Avenue, TH 534G, New York, United States, Fax: 212-263-7254, Tel: 212-263-5072, E-mail: Daniel.ONeill@nyumc.org

Citation: O’Neill DK, Aizer A, Bloom MJ, Kline RP, Chinitz L, et al. (2016) Isoproterenol Increases BIS and Arousal during Catheter Ablation for Atrial Fibrillation. J Anesth Pati Care 1(2): 202. doi: 10.15744/24565490.1 .202

Received Date: June 15, 2016 Accepted Date: August 30, 2016 Published Date: September 02, 2016

\begin{abstract}
Background: Isoproterenol is frequently used to test therapeutic effectiveness post- catheter ablation procedure for atrial fibrillation. Preliminary data suggests that administration of isoproterenol increases the Bispectral index (BIS), implicating the CNS stimulatory effects of isoproterenol.

The authors hypothesize that isoproterenol increases awareness through central nervous system (CNS) stimulatory effects, thereby interfering with a stable neurological state during ablation procedures.

Methods: We administered increasing doses of isoproterenol to twenty patients who were anesthetized with propofol-remifentanil infusions undergoing ablation of atrial fibrillation under general anesthesia. The level of awareness was assessed by BIS, spontaneous or elicited movement, the ability to follow commands, and recall of words repeated to them while under general anesthesia.

Results: After isoproterenol infusion during steady state anesthesia, the BIS value increased significantly in 19 out of 20 patients. The average BIS increase was $24.6 \pm 12.2(\mathrm{P}<0.00001)$.

Twelve (12) patients moved spontaneously and 10 patients followed commands after the isoproterenol, although none of the patients were able to recall words repeated to them or events which occurred while under general anesthesia.

Conclusions: Isoproterenol's CNS stimulatory effects can interfere with general anesthesia during the ablation procedure resulting in patient movement and awareness. The use of additional anesthetic agents during the administration of isoproterenol could be helpful in preventing potentially harmful movement and possible (increase in consciousness) recall in patients having atrial fibrillation ablation procedures under general anesthesia with an isoproterenol challenge.
\end{abstract}

KeyWords: Isoproterenol; BIS; Atrial fibrillation; Ablation; Anesthesia; Electrophysiology 


\section{Introduction}

Atrial fibrillation (AF) is the most common human arrhythmia. Different techniques of AF ablation have been described including probabilistic ones and customized ones [1-5]. Atrial fibrillation ablation procedures are frequently performed with general anesthesia instead of conscious sedation. The use of general anesthesia results in loss of consciousness, amnesia, immobility (areflexia), anti-nociception, and analgesia, all of which are helpful during ablation procedures. One study even suggests that general anesthesia improves the success of ablation procedures compared with conscious sedation.

Isoproterenol can be used within the field of electrophysiology to induce arrhythmias before or after ablation (inducibility testing). This is based on its ability, during ablation procedures for atrial fibrillation, to evoke supraventricular arrhythmias and reveal injured, but electrically viable myocardium, thus revealing aberrant conduction pathways where additional ablation may be necessary. Isoproterenol induces atrial fibrillation in patients with vagotonic, adrenergic and random atrial fibrillation, and is a better predictor of clinical outcome than rapid atrial pacing $[3,4]$.

The stimulatory effects of isoproterenol are due to a direct acting relatively selective Beta-1 and Beta-2 agonist effects, which also lead to non-adrenergic signaling and stimulation of myocardial contractility, bronchodilation, tachycardia, and changes in blood pressure (both hypertension and hypotension). In the awake patient, isoproterenol causes multiple explained that CNS effects such as nervousness, headache, dizziness, restlessness, insomnia, anxiety, tension, blurring of vision, fear, and excitement.

A pilot study $(\mathrm{N}=7)$ by our research group, showed that the bispectral index (BIS) increased dramatically with isoproterenol administration to patients under anesthesia, although no patient had any recall of events [6]. A single patient case report also reported that isoproterenol administration increased the BIS index [7]. In this expanded study, we asked whether patients' level of consciousness was increased, if the subjects moved spontaneously, and whether they could follow commands and recall events following the administration of isoproterenol.

\section{Materials and Methods}

This study was approved by the Institutional Review Board (IRB) of the New York University Langone Medical Center (NYULMC) and was conducted under a Federal Wide Assurance, and in compliance with all relevant OHRP regulations. We enrolled twenty $(\mathrm{N}=20)$ consecutive adult patients with atrial fibrillation who were scheduled for electrophysiology studies and catheter based ablation under total intravenous anesthesia (TIVA-ET). Patients with altered levels of consciousness pre-intervention, or with neuromuscular disease precluding the use of succinylcholine were excluded. The anesthesiologists providing routine patient care were responsible for the placement of the intravenous line, ASA monitors, and general total intravenous anesthesia (TIVAET). All patients received general anesthesia which is standard of care at NYULMC. All patients were positioned supine and monitored according to the ASA monitoring guidelines. Monitoring included measurements of oxygen saturation using pulse oximetry, noninvasive blood pressure, electrocardiogram, capnometry and invasive blood pressure monitoring. General total intravenous anesthesia (TIVA) was induced with propofol, and neuromuscular blocker (succinylcholine or rocuronium). Patients were maintained on infusions of propofol ranging from 25 to $200 \mathrm{mcg} / \mathrm{kg} / \mathrm{min}$ titrated to a BIS target of $50 \pm 5$ and an infusion of remifentanil ranging from 30 to $100 \mathrm{ng} / \mathrm{kg} / \mathrm{min}$ with no further use of muscle relaxants. Controlled ventilation with an endotracheal tube (ET) was maintained with an $\mathrm{ETCO}_{2}=33 \pm 3$ and respiratory rate (RR) [8-12].

The standardized ablation protocol utlitized radiofrequency ablation with an irrigated ablation catheter (Thermocool, Biosense Webster, Diamond Bar, California) combined with the Ensite Velocity Cardiac Mapping System (St. Jude Medical, Saint Paul, Minnesota). Wide area circumferential ablation was performed with one circle encompassing the left pulmonary veins and a second circle encompassing the right pulmonary veins. Entrance block to the pulmonary veins was confirmed utilizing multipole circular mapping catheters. A set of linear lesions was then generated connecting the left superior pulmonary vein to the right superior pulmonary vein. Upon completion of the initial ablation lesions for the procedure and return to sinus rhythm, isoproterenol (4 mcg/ $\mathrm{mL}$ ) was infused using a stepped protocol with $5 \mathrm{~min}$ intervals at $5,10,15$, and $20 \mathrm{mcg} / \mathrm{min}$, similarly to other published studies [13]. Isoproterenol was discontinued in case of induction of a tachycardia with a non-sinus mechanism, hemodynamic instability, ECG changes suggesting ischemia ( $\mathrm{T}$ wave inversions or ST depressions), BIS increase, or patient movements (spontaneous or elicited). BIS values were recorded throughout and a BIS change greater than 5 was defined categorically as a positive BIS response. Propofol and remifentanil infusion levels were kept constant unless spontaneous movement occurred, in which case a rescue bolus of propofol was given to deepen the anesthesia unless extubation was indicated. No catheter manipulation was performed during isoproterenol infusion to minimize any mechanical stimuli to the patient.

A focused intraoperative neurological exam after elimination of neuromuscular blocker (several half-lives after single induction dose) including the ability to follow commands was used to assess the patient's awareness. To assess the patient's ability to follow commands, the anesthesiologist would instruct them to "Squeeze my hand." For assessing the patient's recall, they were asked postoperatively (one hour later) to repeat words spoken while under anesthesia.

At the time words were spoken under anesthesia, spontaneous movements were also tabulated as either present or absent. The neurological exam was performed prior to isoproterenol infusion (time $=0)$, ten $(10)$ minutes later, and at the end of the 
isoproterenol infusion. To assess the patient's recall, the anesthesiologist repeated three sets of words three times each, i.e. at 0 minutes or the start of infusion, at 10 minutes and at 20 minutes. (The first word set was: boat, banana, airplane; the second word set was: train, apple, horse.) The word sets represented forms of transportation, fruit, words beginning with "a" or " $b$ ", and other categories to help retrieval or recall. Patients were asked such questions previously described in the literature [14]. While in the recovery room approximately 1 hour after the procedure or before discharge, whichever was first, to ascertain: (1) What is the last thing you remember before falling asleep? ; (2) What is the first thing you remember after waking up? ; (3) Do you remember any of the words I told you?

\section{Statistics}

Descriptive statistics including means, medians, standard deviations, and ranges were calculated using Excel. Confidence intervals and t-test for paired samples were performed on interval data to demonstrate differences between means. Mean values are presented with \pm one standard deviation. Binomial distribution was used for power analysis. Chi-squared was used to test for significance of nominal data using rates.

\section{Results}

The demographic data for the patients and design summary are shown in Table 1 . Age ranged from 47 to 78 with a mean age $61.9 \pm$ 8.24. There were 15 males and 5 females. Almost half (45\%) of the patients (9/20) had hypertension and $40 \%$ of the patients $(8 / 20)$ were on a beta blocker including two patients on sotolol. Five patients were on dronedarone and one patient was on amiodarone. No patient had a diagnosed altered level of consciousness or severe cognitive impairment although one patient had depression and another had mild Alzheimer's disease.

Consistent with its properties as a beta adrenergic agonist, isoproterenol increased the heart rate in $90 \%$ or 18 out of 20 patients as shown in Table 2A. The mean initial heart rate was $71.5 \pm 22 \mathrm{BPM}$ and the mean heart rate at maximal isoproterenol was 120.9 $\pm 28.2 \mathrm{BPM}$ a statistically significant increase $(\mathrm{P}<0.00001)$. The average heart rate of patients without beta blockers was $64.4 \pm$ $14.8 \mathrm{BPM}$ prior to isoproterenol, whereas the average heart rate with beta blockers was $82.1 \pm 27.4 \mathrm{BPM}$ prior to isoproterenol. Isoproterenol caused the no beta blocker group average heart rate to increase significantly by 57.9 to $122.3 \mathrm{BPM}$ and the beta blocker group to increase significantly by 36.6 to $118.8 \mathrm{BPM}$, but there was no statistically significant difference between the two groups in terms of heart rate effect or baseline heart rate $(\mathrm{p}=0.077)$. The changes in SBP were less predictable; $30 \%$ became hypertensive and $25 \%$ became hypotensive (Table $2 \mathrm{~B}$ ).

\begin{tabular}{|c|c|}
\hline \multicolumn{2}{|c|}{ Baseline Characteristics } \\
\hline Age & Range 47 to 78 years Mean Age $61.9 \pm 8.24$ \\
\hline Sex & Male 15 / Female 5 \\
\hline Preoperative Beta Blocker & $8 / 20=40 \%$ \\
\hline Propofol Dose & $\begin{array}{l}\text { Range } 25 \text { to } 200 \mathrm{mcg} / \mathrm{kg} / \mathrm{min} \\
\text { Mean } 83.4 \pm 32.1 \mathrm{mcg} / \mathrm{kg} / \mathrm{min}\end{array}$ \\
\hline Remifentanil Dose & Range 30 to $100 \mathrm{ng} / \mathrm{kg} / \mathrm{min}$ \\
\hline Maneuver & $\begin{array}{l}\text { Stepwise Infusion Protocol: ( } 5 \text { then } 10 \text { then } 15 \\
\text { then 20) mcg/min q } 5 \text { minute increases }\end{array}$ \\
\hline Isoproterenol Dose & $\begin{array}{l}\text { Range } 16 \text { to } 240 \mathrm{mcg} \\
\text { Mean }=146.8 \pm 77.4\end{array}$ \\
\hline \multirow{5}{*}{$\begin{array}{c}\text { Responses } \\
\text { (associated with termination } \\
\text { of infusion) }\end{array}$} & Heart Rate \\
\hline & Blood Pressure \\
\hline & BIS Change \\
\hline & Movement \\
\hline & Recall \\
\hline
\end{tabular}

Table 1: Demographics and Design Summary

Also shown in Table 2C, four patients had non-sinus arrhythmias in response to the isoproterenol challenge and two patients had ST changes without arrhythmias. Isoproterenol administration was halted for the occurrence of non-sinus arrhythmias (208 mcg, $188 \mathrm{mcg}, 240 \mathrm{mcg}$, and $240 \mathrm{mcg}$ ) and the ST changes (16 mcg and $120 \mathrm{mcg})$. There were three cases of return to atrial fibrillation and one case of a junctional rhythm. The atrial fibrillation patients were cardioverted.

For many of the remaining patients the isoproterenol infusion was halted because of neurological effects as shown in Table 3. The mean BIS increase was $24.6 \pm 12.1$. Figure 1 shows associated spontaneous movement represented by the blue triangles above BIS2 elevations. The difference between the mean BIS pre-isoproterenol and the peak BIS after isoproterenol was statistically significant $(\mathrm{P}<0.00001)$. In all but one of the patients $(95 \%)$, the BIS increased with isoproterenol administration. 


\begin{tabular}{|c|c|c|c|c|}
\hline \multicolumn{5}{|c|}{ A: Heart Rate: Tachycardia 18/20 =90\% } \\
\hline & Pre-Isoproterenol & Post-Isoproterenol & Effect & P Value \\
\hline Overall N = 20 & $71.5 \pm 22$ & $120.9 \pm 28.2$ & +49.4 & $0.00001^{*}$ \\
\hline No Beta Blocker N = 12 & 64.4 & 122.3 & +57.9 & $0.00001^{*}$ \\
\hline Beta Blocker N =8 & 82.1 & 118.8 & +36.7 & $0.00078^{*}$ \\
\hline
\end{tabular}

\begin{tabular}{|c|c|}
\hline \multicolumn{2}{|c|}{ B: Blood Pressure } \\
\hline Effect: SBP2/SBP1 & Frequency \\
\hline Hypertensive: Increase $>20 \%$ & $6 / 20=30 \%$ \\
\hline Normotensive: Same & $9 / 20=45 \%$ \\
\hline Hypotensive: Decrease $>20 \%$ & $5 / 20=25 \%$ \\
\hline
\end{tabular}

\begin{tabular}{|c|c|}
\hline \multicolumn{2}{|c|}{ C: Arrhythmia } \\
\hline Atrial Fibrillation Requiring Cardioversion & $3 / 20=15 \%$ \\
\hline Junctional Rhythm & $1 / 20=5 \%$ \\
\hline ST Segment Changes & $2 / 20=10 \%$ \\
\hline
\end{tabular}

Table 2: Cardiovascular Effects

\begin{tabular}{|c|c|c|c|c|}
\hline & $\begin{array}{c}\text { BIS Change: } \\
\text { Frequency }\end{array}$ & $\begin{array}{c}\text { Mean BIS } \\
\text { Change }\end{array}$ & $\begin{array}{c}\text { Spontaneous } \\
\text { Movement }\end{array}$ & $\begin{array}{c}\text { Follows } \\
\text { Commands }\end{array}$ \\
\hline Overall & $19 / 20=95 \%$ & $24.6 \pm 12.2^{*}$ & $12 / 20=60 \%$ & $10 / 20=50 \%$ \\
\hline No Beta Blocker & $12 / 12=100 \%$ & 28.0 & $9 / 12=75 \%$ & $8 / 12=66.7 \%$ \\
\hline Beta Blocker & $7 / 8=87.5 \%$ & 19.5 & $3 / 8=37.5 \%$ & $2 / 8=25.0 \%$ \\
\hline
\end{tabular}

${ }^{\star}$ Paired student's t test. $\mathrm{p}<0.00001$

Recall: $0 / 20=0 \%$

Table 3: Neurological Effects

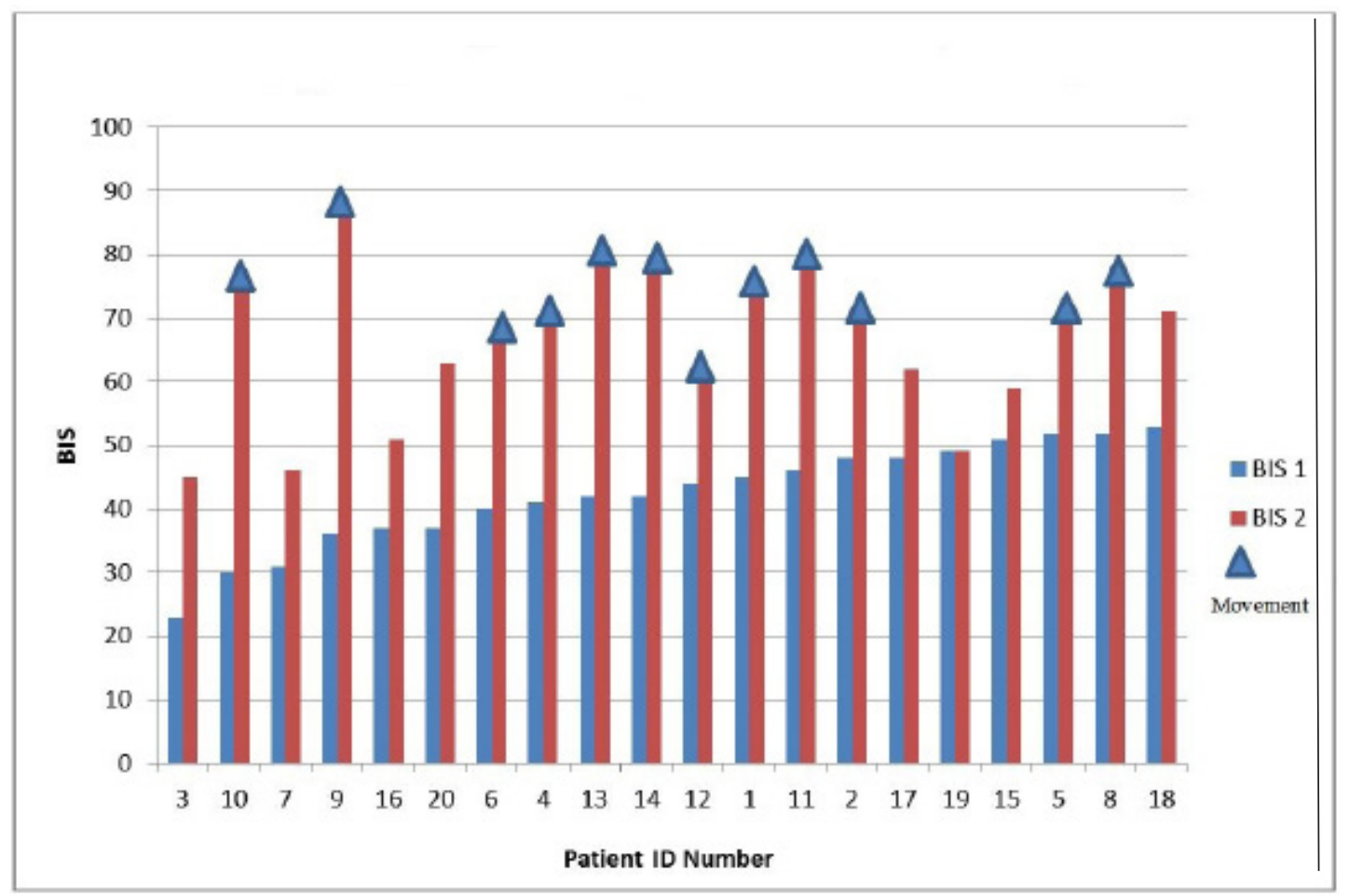

Figure 1: BIS Change after Isoproterenol

There was no significant difference in average propofol infusion rates for patients who were on preoperative beta blockers vs. those who were not. The one patient (Subject 19) was on a standard dose of a beta blocker which could counteract the effects of isoproterenol on the central nervous system by passing the blood brain barrier. 
the risk of recall with isoproterenol vs. without isoproterenol estimated to be one in 1000 or one in 10,000 in the usual general anesthesia population $[9,15]$.

\section{Measurement of Arousal}

Though the underlying CNS mechanisms of isoproterenol are complex, in this study, we monitored these effects by several standard metrics including BIS levels, patient ability to follow commands, and patient recall. This study utilized a formal isoproterenol infusion protocol and controlled mechanical ventilation. In the pilot study [6] the median isoproterenol dose was 25 mcg with arbitrary dosing, spontaneous ventilation and mild hypercarbia, this study with the isoproterenol infusion protocol had a mean isoproterenol dose of $146.8 \mathrm{mcg}$ with normocarbia.

This suggests that a relationship between carbon dioxide and isoproterenol sensitivity in the brain analogous to the apneic threshold, but a dose response relationship was not measured [16]. The BIS Vista ${ }^{\mathrm{TM}}$ Monitor is a non-invasive device that measures the electrical activity of the brain. Some studies suggest that using the BIS value to guide administration of anesthetic medications results in increased accuracy of therapeutic dosages [8]. In contrast, another study did not find any difference between BIS guided anesthetic dosing and use of inhalational agent [9].

Moreover, BIS values do not change in a linear fashion with increasing anesthetic dose [10]. Some studies suggested that a low BIS value during a general anesthetic was associated with a higher morbidity and mortality although this has been called into question when co-morbidities are taken into account [11,12]. Alternatively, a high BIS value is associated with spontaneous movement, awareness and recall. Despite these uncertainties, our combination of monitoring approaches gives us important clinical information as to changes in awareness to avoid undesirable outcomes.

\section{Evidence for interaction of adrenergics with arousal}

Agents which act on the adrenergic system have also been shown to affect the level of arousal in anesthetized subjects. In a case report, ephedrine, an indirect acting alpha and beta agonist, was shown to increase BIS values although phenylephrine had no effect [17]. Atenolol, landiolol and esmolol all attenuate BIS, heart rate and BP responses to intubation. Metoprolol and clonidine (alpha-2 agonist sympatholytic) decrease propofol requirements during BIS titrated anesthesia. Esmolol reduces the amount of anesthesia required to prevent movement at skin incision [18-21]. In anesthetized rats, propanolol and esmolol cause burst suppression and changes in the EEG suggestive of non REM sleep. Studies using radiographically tagged beta blockers in rats and PET scans after adrenergic stimulation in humans suggest that the location of adrenergic receptors in the brain is widespread $[22,23]$. Beta receptors are found in the pontine reticular formation, the hippocampus, the hypothalamus, the thalamus, the cerebellum and multiple locations in the cortex [24,25]. These adrenergic receptors are targeted by norepinephrine releasing projections from brainstem cell clusters known as the locus coeruleus (A6) and A1, A2, A3, A4, A5 and A7. The locus coeruleus alone has five major projections. In anesthetized rats, application of norepinephrine to the medial septal area or the medial preoptic area, both of which receive projections from the locus coeruleus, results in behavioral and EEG measured arousal [26]. These results suggest that targets of the locus coeruleus may be involved in the arousal seen with administration of isoproterenol.

If isoproterenol had a single site of action, we might expect to see a threshold effect of isoproterenol dose on patient arousal. We might also expect that the arousal would occur at similar doses of isoproterenol in each patient. We saw patient arousal at a wide range of isoproterenol dosages indicating plasma drug concentration variations which may be a function of the level of anesthesia or plasma propofol concentration prior to isoproterenol administration.

However, we speculate that the effects of isoproterenol on patient arousal may be happening at different sites in the brain. Increasing inhibition by arousal centers such as the locus coeruleus on the ventrolateral preoptic nucleus (VLPO) nucleus and other sleep centers could cause a shift from unconsciousness to awareness. Direct stimulatory action on the cortex and indirect activation of thalamic nuclei could activate pathways involved in learning, memory and awareness.

\section{Monitoring arousal and the significance of measured BIS changes}

We used other approaches to assess patient arousal including the patient's ability to follow commands. This response has been shown to occur at BIS levels as low as 40 although the patients had no recall of these events [27]. Another approach to evaluating the level of consciousness of anesthetized patients is to assess their ability to form memories or recall events which occurred while under anesthesia. A target BIS of 40 to 60 is recommended by the manufacturers to be sufficient to prevent recall. Indeed, various studies support the lack of conscious recall in the vast majority of patients at a BIS less than $60[28,29]$. We tested for conscious recall of words repeated to the patients while under anesthesia and for recall of events occurring while under anesthesia. Although there were no episodes of recall, the BIS response especially in combination with the examples of spontaneous movement and ability to follow commands suggests that isoproterenol significantly promoted arousal during steady state general anesthesia.

\section{Conclusions}

This study demonstrates an increase in arousal of anesthetized patients during isoproterenol administration for atrial ablation procedures as measured by BIS level, movement and the ability to follow commands. Our data suggest that use of additional anesthetic agents during the administration of isoproterenol could be helpful in preventing harmful movement and possible 
recall. BIS may be a useful guide in the optimization of anesthesia when isoproterenol is administered during electrophysiology procedures.

\section{Summary}

1. Isoproterenol increased BIS values in 19/20 subjects.

2. The confidence interval for the mean BIS increase is 19.3 to 29.9 , which is highly significant.

3. The patients who demonstrated a BIS response tend to move spontaneously $(12 / 19=63.2 \%)$ and have the ability to follow simple commands $(10 / 19=52.6 \%)$ despite their continued amnesia (recall rate $=0 \%)$.

4. Clinically, the isoproterenol infusion to access the quality of AF ablation poses several anesthetic risks including movement effects on mapping, awareness during cardioversion, hemodynamic instability, and prolonged mechanical ventilation secondary to possible dosing of neuromuscular blockade at the end of the case.

5. With the understanding that the isoproterenol infusion can increase arousal during the last 20 to 30 minutes of the catheter ablation procedure, the anesthesia provider may choose to compensate by carefully increasing the dose of short acting anesthetic agents such as proprofol and/or remifentanil and avoid the prolonged action of neuromuscular blockers.

\section{Acknowledgement}

Neil Bernstein, M.D., Douglas Holmes, M.D., Scott Bernstein, M.D., Mark Mascarenhas, M.D., Steven Fowler, M.D., Aileen Ferrick, ACNP, FHRS, Melissa Hull, MD,Ph.D, Jane Smyth-Melsky, RN, Marianne Paruch RN, MBA, Michael Haile, M.D., Lola Franco, Denise Sennett, R.N., Amanda Sacks, Ph.D. Andrew Rosenberg, M.D.

(https://www.clinicaltrials.gov/ct2/show/NCT01377636?term=isoproterenol\&rank=1)

Institutional Support: New York University School of Medicine

\section{Meetings}

International Anesthesia Research Society (IARS) May 21 to 24, 2011, Vancouver, Canada.

Heart Rhythm Society (HRS) May 4 to 7, 2011, San Francisco, CA

\section{References}

1. Oral H, Pappone C, Chugh A, Good E, Bogun F, et al. (2006) Circumferential pulmonary-vein ablation for chronic atrial fibrillation. N Engl J Med 354: 934-41. 2. Ernst S, Ouyang F, Löber F, Antz M, Kuck KH (2003) Catheter-induced linear lesions in the left atrium in patients with atrial fibrillation: an electroanatomic study. J Am Coll Cardiol 42: 1271-82.

3. Nademanee K, McKenzie J, Kosar E, Schwab M, Sunsaneewitayakul B, et al. (2004) A new approach for catheter ablation of atrial fibrillation: mapping of the electrophysiologic substrate. J Am Coll Cardiol 43: 2044-53.

4. Seitz J, Bars C, Ferracci A, Maluski A, Penaranda G, et al. (2016) Electrogram Fractionation-Guided Ablation in the Left Atrium Decreases the Frequency of Activation in Pulmonary Veins and Leads to Atrial Fibrillation Termination. JACC doi:10.1016/j.jacep.2016.04.003.

5. Narayan SM, Krummen DE, Shivkumar K, Clopton P, Rappel WJ, et al. (2012) Treatment of atrial fibrillation by the ablation of localized sources: CONFIRM (Conventional Ablation for Atrial Fibrillation With or Without Focal Impulse and Rotor Modulation) trial. J Am Coll Cardiol 60: 628-36.

6. O'Neill DK, Aizer A, Linton P, Bloom M, Rose E, et al. (2012) Isoproterenol increases level of consciousness during catheter ablation of atrial fibrillation. J Interv Card Electrophysiol 34: 137-42.

7. Matthews R (2006) Isoproternol-induced elevated bispectral indexes while undergoing radiofrequency ablation: A case report. AANA J 74: 193-5.

8. Myles PS, Leslie K, McNeil J, Forbes A, Chan MT, et al. (2004) Bispectral index monitoring to prevent awareness during anaesthesia: The B-aware randomized controlled trial. Lancet 363: 1757-63.

9. Avidan MS, Zhang L, Burnside BA, Finkel KJ, Searleman AC, et al. (2008) Anesthesia awareness and the bispectral index”. N Engl J Med 358: $1097-108$.

10. Kent CD, Domino KB (2009) Depth of anesthesia. Curr Opin Anaesthesiol 22: 782-7.

11. Monk TG, Saini V, Weldon BC, Sigl JC (2005) Anesthetic management and one year mortality after non-cardiac surgery. Anesth Analg 100: 4-10.

12. Kertai MD, Palanca BJ, Pal N, Burnside BA, Zhang L, et al. (2011) Bispectral index monitoring, duration of bispectral index below 45, patient risk factors, and intermediate-term mortality after noncardiac surgery in the B-unaware trial. Anesthesiol 114: 545-56.

13. Chihrin SM, Mohamed U, Yee R, Gula LJ, Klein GJ, et al. (2008) Utility of Isoproterenol in Unmasking Latent Escape Rhythm in Pacemaker Dependent Patients Undergoing Pacemaker Replacement. Am J Cardiol 101: 631-3.

14. Nordström O, Sandin R (1996) Recall during intermittent propofol anaesthesia. Br J Anaesth 76: 699-701.

15. Kerssens C, Klein J, Bonke B (2003) Awareness: Monitoring versus remembering what happened. Anesthesiol 99: 570-5.

16. Boden AG, Haris MC, Parker MJ (1985) Apneic threshold for CO2 in the anesthetized rat: fundamental properties under steady-state conditions. J Appl Physiol 85: 898-907.

17. Shiyama T, Oguchi T, Iijima T, Matsukawa T, Kashimoto S, et al. (2003) Ephedrine, but not phenylephrine, increases bispectral index values during combined general and epidural anesthesia. Anesth Analg 97: 780-4.

18. Yamakage M, Sasaki H, Mizuuchi M, Iwasaki S, Namiki A (2004) Effects of oral atenolol on volatile anesthetic induction with sevoflurane in adults. J Anaesth 18: $185-9$. 
19. Oda Y, Nishikawa K, Hase I, Asada A (2005) The Short-Acting B1-Adrenoceptor Antagonists Esmolol and Landiolol Suppress the Bispectral Index Response to Tracheal Intubation During Sevoflurane Anesthesia. Anesth Analg 100: 733-7.

20. Fehr SB, Zalunardo MP, Seifert B (2001) Clonidine decrease propofol requirements during anaesthesia: Effect on bispectral index. Br J Anaesth 86: 627-32.

21. Johansen JW, Flaishon R, Sebel PS (1997) Esmolol reduces anesthetic requirement for skin incision during propofol/nitrous oxide/morphine anesthesia. Anesthesiol 86: 364-71.

22. Kurita T, Takata K, Morita K, Sato S (2008) Lipophilic $\beta$-adrenoceptor antagonist propranolol increases the hypnotic and anti-nociceptive effects of isoflurane in a swine model. Br J Anaesth 100: 841-5.

23. Johansen JW (2001) Esmolol promotes electroencephalographic burst suppression during propofol/alfentanil anesthesia. Anesth Analg 93: 1526-31.

24. Cameron OG, Minoshima S (2002) Regional brain activation due to pharmacologically induced adrenergic interoceptive stimulation in humans. Psychosom Med 64: 851- 61.

25. Rainbow TC, Parsons B, Wolfe BB (1984) Quantitative autoradiography of B1- and B2- adrenergic receptors in rat brain. Proc Natl Acad Sci U S A 81: 1585-9.

26. Berridge CW, O’Neill J (2001) Differential sensitivity to the wake promoting actions of norepinephrine within the medial preoptic area and the substantia innominate. Behav Neurosci 115: 165-74.

27. Kocoman AB, Demiraran Y, Yalcin SG, Akcali G, Somunkiran A (2007) Use of the bispectral index to predict a positive awareness reaction to laryngeal mask airway-Fastrach insertion and intubation. Acta Anaesthesiol Scand 51: 1368-72.

28. Kerssens C, Gaither JR, Sebel PS (2009) Preserved memory function during bispectral index- guided anesthesia with sevoflurane for major orthopedic surgery. Anesthesiology 111: 518-24.

29. Dahhaba AA (2005) Different conditions that could result in the Bispectal Index indicating an incorrect hypnotic state. Anesth Analg 101: $765-73$.

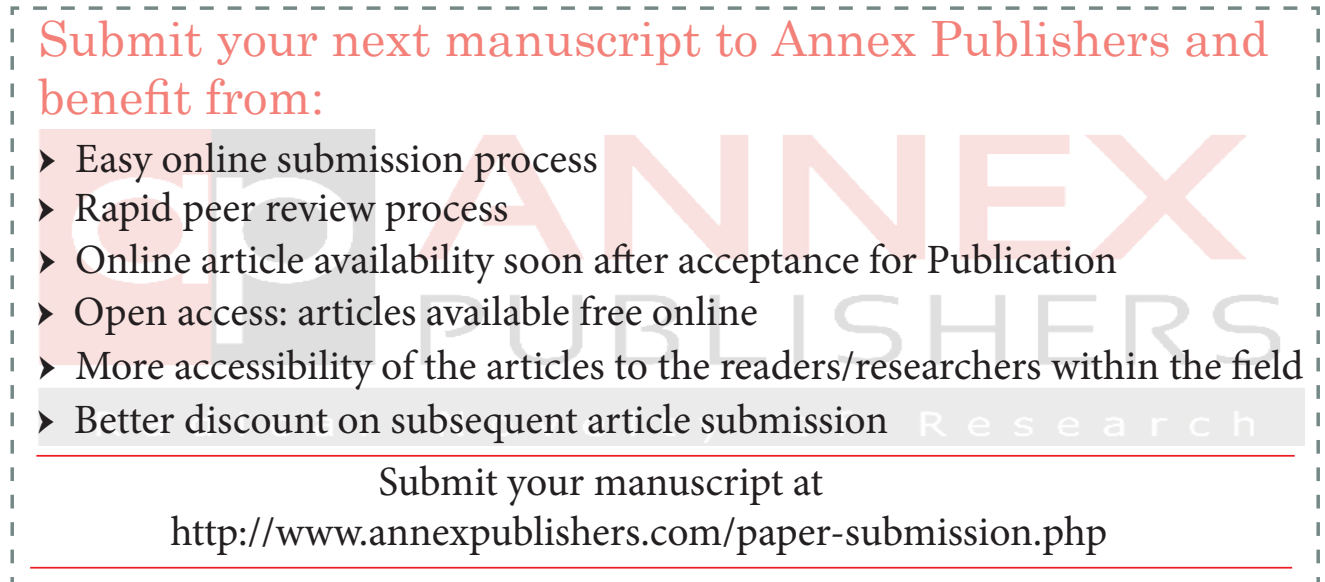

